\title{
Idempotents in an ultrametric Banach algebra
}

\author{
Alain Escassut \\ Université Clermont Auvergne, \\ UMR CNRS 6620, LMBP, \\ F-63000 Clermont-Ferrand, France. \\ alain.escassut@uca.fr
}

\begin{abstract}
Let $\mathbb{K}$ be a complete ultrametric field and let $A$ be a unital commutative ultrametric Banach $\mathbb{K}$-algebra. Suppose that the multiplicative spectrum admits a partition in two open closed subsets. Then there exist unique idempotents $u, v \in A$ such that $\phi(u)=1, \phi(v)=0 \forall \phi \in$ $U, \phi(u)=0 \phi(v)=1 \forall \phi \in V$. Suppose that $\mathbb{K}$ is algebraically closed. If an element $x \in A$ has an empty annulus $r<|\xi-a|<s$ in its spectrum $s p(x)$, then there exist unique idempotents $u, v$ such that $\phi(u)=1, \phi(v)=0$ whenever $\phi(x-a) \leq r$ and $\phi(u)=0, \phi(v)=1$ whenever $\phi(x-a) \geq s$.
\end{abstract}

\section{RESUMEN}

Sea $\mathbb{K}$ un cuerpo ultramétrico completo y sea $A$ una $\mathbb{K}$-algebra de Banach ultramétrica unital conmutativa. Suponga que el espectro multiplicativo admite una partición en dos conjuntos abiertos y cerrados. Luego, existen idempotentes únicos $u, v \in A$ tales que $\phi(u)=1, \phi(v)=$ $0 \forall \phi \in U, \phi(u)=0 \phi(v)=1 \forall \phi \in V$. Suponga que $\mathbb{K}$ es algebraicamente cerrado. Si un elemento $x \in A$ tiene un anillo vacío $r<|\xi-a|<s$ en su espectro $s p(x)$, entonces existen idempotentes únicos $u, v$ tales que $\phi(u)=1, \phi(v)=0$ cada vez que $\phi(x-a) \leq r$ y $\phi(u)=0, \phi(v)=1$ cada vez que $\phi(x-a) \geq s$.

Keywords and Phrases: ultrametric Banach algebras, multiplicative semi-norms, idempotents, affinoid algebras.

2020 AMS Mathematics Subject Classification: 12J25, 30D35, 30 G06.

\section{(cc) BY-NC}




\section{Introduction and main Theorem}

Ultrametric Banach algebras have been a topic of many resarch along the last years [1], [3], [4], [5],[6], [10], [11], [12]. The following Theorem 1.1 (stated in [14]) corresponds in ultrametric Banach algebras to a well known theorem in complex Banach algebra: if the spectrum of maximal ideals admits a partition in two open closed subsets $U$ and $V$ with respect to the Gelfand topology, there exist idempotents $u$ and $v$ such that $\chi(u)=1, \chi(v)=0 \forall \chi \in U$ and $\chi(u)=0, \chi(v)=1 \forall \chi \in V$.

In an ultrametric Banach algebra, it is impossible to have a similar result because a partition in two open closed subsets for the Gelfand topology on the spectrum of maximal ideals then makes no sense, due to the total disconnection of the spectrum. B. Guennebaud first had the idea to consider the set of continuous multiplicative semi-norms of an ultrametric Banach algebra, denoted by $\operatorname{Mult}(A,\|\|$.$) instead of the spectrum of maximal ideals [14], an idea that later$ suggested Berkovich theory [2]. Recall that $\operatorname{Mult}(A,\|\|$.$) is compact with respect to the topology$ of pointwise convergence (Theorem 1.11 in [7]).

The proof of Theorem 1.1, stated in [14], was heavy and involved many particular notions in a chapter of over 40 pages that was never published. We will use Propositions 2.10, 2.11, 2.12 in order to assure the unicity. Finally, we will show that if the theorem is proven for affinoid algebras, that may be generalised to all ultrametric Banach algebras (Proposition 2.12).

Notations: We denote by $\mathbb{K}$ a complete ultrametric field. Given a $\mathbb{K}$-algebra $A$, we denote by $\operatorname{Mult}(A)$ the set of multplicative semi-norms of $A$ and if $A$ is a normed $\mathbb{K}$-algebra, we denote by $\operatorname{Mult}(A,\|\|$.$) the set of continuous multplicative semi-norms of A$ provided with the topology of pointwise convergence. Next, we denote by $\operatorname{Mult}_{m}(A,\|\|$.$) the set of continuous multplicative$ semi-norms of $A$ whose kernel is a maximal ideal of $A$. Given $\phi \in \operatorname{Mult}(A,\|\cdot\|)$, we denote by $\operatorname{Ker}(\phi)$ the closed prime ideal of the $x \in A$ such that $\phi(x)=0$.

It is well known that every maximal ideal is the kernel of at least one multiplicative semi-norm on $A$ (see for example [9]). The algebra $A$ is said to be multbijective if for every maximal ideal $\mathcal{M}$, $\frac{A}{\mathcal{M}}$ admits only one absolute value that is an expansion of this of $\mathbb{K}$. It is easily seen that if every maximal ideal is of finite codimension, then the algebra $A$ is multbijective.

Consider then a multbijective unital commutative ultrametric $\mathbb{K}$-Banach algebra $A$. We denote by $\mathcal{X}(A)$ the set of algebra homomorphisms from $A$ onto a field extension of $\mathbb{K}$ of the form $\frac{A}{\mathcal{M}}$ where $\mathcal{M}$ is a maximal ideal of $A$. So, for every $\chi \in \mathcal{X}(A)$, the mapping $|\chi|$ defined on $A$ by $|\chi|(x)=|\chi(x)|$ belongs to $\operatorname{Mult}_{m}(A,\|\|$.$) and this is the unique \phi \in \operatorname{Mult}_{m}(A,\|\|$.$) such that$ $\operatorname{Ker}(\phi)=\operatorname{Ker}(\chi)$.

Theorem 1.1. Let $A$ be a unital commutative ultrametric $\mathbb{K}$-Banach algebra such that $\operatorname{Mult}(A,\|\cdot\|)$ admits a partition in two compact subsets $U, V$. There exist unique idempotents $u, v \in A$ such that $\phi(u)=1, \phi(v)=0, \forall \phi \in U$ and $\phi(u)=0, \phi(v)=1, \forall \phi \in V$. 
Corollary 1.2. Let $A$ be a unital commutative ultrametric $\mathbb{K}$-Banach algebra such that Mult $(A,\|\cdot\|)$ admits a partition in two compact subsets $U, V$. Then $A$ is isomorphic to a direct product of two $\mathbb{K}$-Banach algebras $A_{U} \times A_{V}$ such that $\operatorname{Mult}\left(A_{U},\|\cdot\|\right)=U$ and $\operatorname{Mult}\left(A_{V},\|\cdot\|\right)=V$. Given the idempotent $u \in A$ such that $\phi(u)=1 \forall \phi \in U, \phi(u)=0 \forall \phi \in V$, then $A_{U}=u A, A_{V}=(1-u) A$.

As an easy consequence, we have Theorem 1.3. A few definitions are necessary:

Definitions and notations: Suppose that $\mathbb{K}$ is algebraically closed. Let $a \in \mathbb{K}$ and $r, s \in \mathbb{R}_{+}$ with $0<r<s$. We denote by $\Gamma(a, r, s)$ the set $\{x \in \mathbb{K}|r<| x-a \mid<s\}$. Let $D$ be a subset of $\mathbb{K}$, let $a \in D$ be such that $D \cap \Gamma(a, r, s)=\emptyset$ and that $r=\sup \{|a-x|, x \in D,|a-x| \leq r\}$ and $s=\inf \{|a-x|, x \in D,|a-x| \geq s\}$. The annulus $\Gamma(a, r, s)$ is called an empty-annulus of $D$.

Let $A$ be a unital commutative $\mathbb{K}$-algebra and let $x \in A$. We denote by $s p(x)$ the set of all $\lambda \in \mathbb{K}$ such that $x-\lambda$ is not invertible.

Theorem 1.3. Suppose that $\mathbb{K}$ is algebraically closed. Let $A$ be a unital commutative ultrametric $\mathbb{K}$-Banach algebra such that $\operatorname{Mult}_{m}(A, \|$. $\|)$ is dense in $\operatorname{Mult}(A,\|\|$.$) and let x \in A$ be such that $s p(x)$ admits an empty-annulus $\Gamma(a, r, s)$. Then there exist a unique idempotent $u \in A$ and $a$ unique idempotent $v \in A$ such that $\chi(u)=1, \chi(v)=0 \forall \chi \in \mathcal{X}(A)$ satisfying $|\chi(x)-a| \leq r$ and $\chi(u)=0, \chi(v)=1 \forall \chi \in \mathcal{X}(A)$ satisfying $|\chi(x)-a| \geq s$.

\section{The proofs}

Proving theorem 1.1 requires some preparation. We will use Propositions 2.10, 2.11 and 2.12 and mainly Theorem 2.7 .

Definitions and notations: Let $A$ be a unital commutative ultrametric $\mathbb{K K}$-Banach algebra whose norm is $\|\cdot\|$. We define the spectral semi-norm $\|\cdot\|_{s p}$ as $\|f\|_{s p}=\lim _{n \rightarrow+\infty}\left\|f^{n}\right\|^{\frac{1}{n}}$. By [13] we have Theorem 2.1 (see also [9], theorem 6.19).

Theorem 2.1. $\|f\|_{s p}=\sup \{\phi(f) \mid \phi \in \operatorname{Mult}(A,\|\cdot\|)\}$.

Affinoid algebras were introduced by John Tate in [17] who called them algebras topologically of finite type and are now usually called affinoid algebras. As this first name suggests, such an algebra is the completion of an algebra of finite type for a certain norm.

Definitions and notation: The $\mathbb{K}$-algebra of polynomials in $n$ variables $\mathbb{K}\left[X_{1}, \ldots, X_{n}\right]$ is equipped with the Gauss norm $\|$. $\|$ defined as

$$
\left\|\sum_{i_{1}, \ldots, i_{n}} a_{i_{1}, \ldots, i_{n}} X_{1}^{i_{1}} \cdots X_{n}^{i_{n}}\right\|=\sup _{i_{1}, \ldots, i_{n}}\left|a_{i_{1}, \ldots, i_{n}}\right| .
$$


We denote by $\mathbb{K}\left\{X_{1}, \ldots, X_{n}\right\}$ the set of power series in $n$ variables $\sum_{i_{1}, \ldots, i_{n}} a_{i_{1}, \ldots, i_{n}} X_{1}^{i_{1}} \cdots X_{n}^{i_{n}}$ such that $\lim _{i_{1}+\ldots+i_{n} \rightarrow \infty} a_{i_{1}, \ldots, i_{n}}=0$. The elements of such an algebra are called the restricted power series in $n$ variables, with coefficients in $\mathbb{K}$. Hence, by definition, $\mathbb{K}\left[X_{1}, \ldots, X_{n}\right]$ is dense in $\mathbb{K}\left\{X_{1}, \ldots, X_{n}\right\}$. Then $\mathbb{K}\left\{X_{1}, \ldots, X_{n}\right\}$ is a $\mathbb{K}$-Banach algebra which is just the completion of $\mathbb{K}\left[X_{1}, \ldots, X_{n}\right]$ and is denoted by $T_{n}$.

By [16] (see also [9]): we have Theorem 2.2:

Theorem 2.2. Every algebra $\mathbb{K}\left\{X_{1}, \ldots, X_{n}\right\}$ is factorial and all ideals are closed.

A $\mathbb{K}$ - affinoid algebra corresponds to a quotient of any algebra of the form $\mathbb{K}\left\{X_{1}, \ldots, X_{n}\right\}$ by one of its ideals equipped with its quotient norm of Banach $\mathbb{K}$-algebra.

By Theorems 31.1 and 32.7 of [9] (see also [17] and [14]):

Theorem 2.3. Let $A$ be a $\mathbb{K}$-affinoid algebra. Then $A$ is noetherian and all its ideals are closed. Each maximal ideal is of finite codimension. Moreover the nilradical of $A$ is equal to its Jacobson radical. Further, A has finitely many minimal prime ideals.

By Theorems 35.4 in [9] or Proposition 2.8 of III in [14], we have Theorem 2.4:

Theorem 2.4. Let $A$ be a $\mathbb{K}$-affinoid algebra. Then $\operatorname{Mult}_{m}(A,\|\|$.$) is dense in \operatorname{Mult}(A,\|\|$. for the topology of pointwise convergence.

By Theorems 35.4 in [9] we can state Theorem 2.5:

Theorem 2.5. Let $A$ be a reduced $\mathbb{K}$-affinoid algebra. Then the spectral norm $\|$. $\|$ of $A$ is a norm and is equivalent to the norm of affinoid algebra.

Remark 2.6. The proofs given in [9] for Theorems 2.2, 2.3, 2.4, 2.5 are given for algebraically closed complete ultrametric field but they hold on any complete ultrametric field.

By Corollary 2.2.7 in [2] we have Theorem 2.7:

Theorem 2.7. Let $A$ be a reduced $\mathbb{K}$-affinoid algebra such that $M$ ult $(A,\|\cdot\|)$ admits a partition in two compact subsets $U_{1}$ and $U_{2}$. Then $A$ is isomorphic to a direct product $A_{1} \times A_{2}$ where $A_{j}$ is a $\mathbb{K}$-affinoid algebra such that Mult $\left(A_{j}, \|\right.$. \|) is homeomorphic to $U_{j}, j=1,2$.

Proposition 2.8. Let $A$ be a $\mathbb{K}$-affinoid algebra of Jacobson radical $\mathcal{R}$ and let $w \in \mathcal{R}$. The equation $x^{2}-x+w=0$ has a solution in $\mathcal{R}$.

Proof. Since $A$ is affinoid, by Theorem 2.3, $w$ is nilpotent, hence we can consider the element

$$
u=-\frac{1}{2} \sum_{n=1}^{+\infty}\left(\begin{array}{c}
\frac{1}{2} \\
n
\end{array}\right)(-4 w)^{n} .
$$


Now we can check that $(2 u-1)^{2}=1-4 w$ and then $u^{2}-u-w=0$.

Proposition 2.9. Let $A$ be a $\mathbb{K}$-affinoid algebra of Jacobson radical $\mathcal{R}$ and let $w \in A$ be such that $w^{2}-w \in \mathcal{R}$. There exists an idempotent $u \in A$ such that $w-u \in \mathcal{R}$.

Proof. We will roughly follow the proof known in complex algebra [15]. Let $r=w^{2}-w$. We first notice that $1+4 r=(2 w-1)^{2}$. Next, $\frac{r}{1+4 r}$ belongs to $\mathcal{R}$ hence by Proposition 2.8, there exists $x \in \mathcal{R}$ such that $x^{2}-x+\frac{r}{1+4 r}=0$, and hence

$$
((2 w-1) x)^{2}-(2 w-1)^{2} x+r=0 .
$$

Now set $s=(2 w-1) x$. Then $s$ belongs to $\mathcal{R}$, as $x$. Then we obtain

$$
s^{2}-(2 w-1) s+r=0 .
$$

Let us now put $u=w-s$ and compute $u^{2}$ :

$$
(w-s)^{2}=w^{2}-2 w s+s^{2}=w+r-2 w s+s^{2} .
$$

But $s^{2}=-r+(2 w-1) s$, hence finally:

$$
(w+s)^{2}=w-r+2 w s+r-(2 w-1) s=w+s .
$$

Thus $u$ is an idempotent such that $u-w \in \mathcal{R}$.

Proposition 2.10. [14] Let $A$ be a commutative unital ultrametric $\mathbb{K}$-Banach algebra and assume that $\operatorname{Mult}(A,\|\|$.$) admits a partition in two compact subsets U, V$. Suppose that there exist two idempotents $u$ and $e$ such that $\forall \phi \in U, \phi(u)=\phi(e)=1$ and $\forall \phi \in V, \phi(u)=\phi(e)=0$. Then $u=e$.

Proof. Put $e=u+r$. Since $e^{2}=e$, we have $(u+r)^{2}=u+2 u r+r^{2}$ hence $u+r=u+2 u r+r^{2}$ and hence $r=2 u r+r^{2}$, therefore $r(2 u+r-1)=0$.

Suppose $r \neq 0$. Then $2 u+r-1$ is a divisor of zero. Now, when $\phi \in U$, we have $\phi(1-u)=0$, hence $\phi(-1+2 u+r)=\phi(u+r)=\phi(e)=1$, and when $\phi \in V$, we have $\phi(u)=\phi(e)=0$, hence $\phi(1-2 u-r)=\phi(1-u-r)=\phi(1-e)=1$. Hence, $\forall \phi \in \operatorname{Mult}(A,\|\cdot\|)$, we have $\phi(1-2 u-r)=1$. Consequently, $1-2 u-r$ does not belong to any maximal ideal of $A$ and hence is invertible. But then $1-2 u-r$ is not a divisor of zero, which proves that $r=0$ and hence $e=u$.

Proposition 2.11. [14] Let $A$ be a $\mathbb{K}$-affinoid algebra such that Mult $(A,\|\cdot\|)$ admits a partition in two compact subsets $U_{1}, U_{2}$. There exist unique idempotents $e_{1}, e_{2} \in A$ such that $\phi\left(e_{1}\right)=$ $1, \phi\left(e_{2}\right)=0 \forall \phi \in U_{1}$ and $\phi\left(e_{1}\right)=0, \phi\left(e_{2}\right)=1 \forall \phi \in U_{2}$. 
Proof. Suppose first that $A$ is reduced. By Theorem 2.7, $A$ is isomorphic to the direct product $A_{1} \times A_{2}$ where $A_{j}$ is a $\mathbb{K}$-affinoid algebra such that $\operatorname{Mult}\left(A_{j},\|\|.\right)=U_{j}, j=1,2$. Let $\Phi$ be the isomorphism from $A_{1} \times A_{2}$ onto $A$, let $u_{j}$ be the unity of $A_{j}, j=1,2$ and let $e_{1}=$ $\Phi\left(u_{1}, 0\right), e_{2}=\Phi\left(0, u_{2}\right)$. So $e_{1}, e_{2}$ are idempotents of $A$. Let $A_{1}^{\prime}=\left\{\Phi(x, 0) \mid x \in A_{1}\right\}$ and let $A_{2}^{\prime}=\left\{\Phi(0, x) \mid x \in A_{2}\right\}$.

Then, given $\varphi \in U_{j}$, it factorizes in the form $\psi \circ \Phi^{-1}$ with $\psi \in \operatorname{Mult}\left(A_{j},\|\cdot\|\right),(j=1,2)$ and for $\varphi \in U_{1}$, we have $\varphi\left(e_{1}\right)=1, \varphi\left(e_{2}\right)=0$, and given $\varphi \in U_{2}$, we have $\varphi\left(e_{1}\right)=0, \varphi\left(e_{2}\right)=1$. By Proposition 2.10, the idempotents $e_{1}, e_{2}$ are unique.

We can easily greneralize when $A$ is no longer supposed to be reduced. Let $\mathcal{R}$ be the Jacobson radical of $A$ and let $B=\frac{A}{\mathcal{R}}$. Let $\theta$ be the canonical surjection from $A$ onto $B$. Every $\phi \in$ $\operatorname{Mult}(A,\|\cdot\|)$ is of the form $\varphi \circ \theta$ with $\varphi \in \operatorname{Mult}(B,\|\cdot\|)$. Let $U_{1}^{\prime}=\{\varphi \in \operatorname{Mult}(B,\|\|)$.$\} be$ such that $\varphi \circ \theta \in U_{1}$ and let $U_{2}^{\prime}=\{\varphi \in \operatorname{Mult}(B,\|\|)$.$\} be such that \varphi \circ \theta \in U_{2}$. Then $U_{1}^{\prime}$ and $U_{2}^{\prime}$ are two compact subsets making a partition of $\operatorname{Mult}(B,\|\cdot\|)$. Therefore, $B$ has an idempotent $u_{1}$ such that $\varphi\left(u_{1}\right)=1 \forall \varphi \in U_{1}^{\prime}$ and $\varphi\left(u_{1}\right)=0 \forall \varphi \in U_{2}^{\prime}$. Let $w \in A$ be such that $\theta(w)=u_{1}$. Then we can check that $\phi(w)=1 \forall \phi \in U_{1}$ and $\phi(w)=0 \forall \phi \in U_{2}$. But by Proposition 2.9, there exists an idempotent $e_{1} \in A$ such that $e_{1}-w \in \mathcal{R}$. Then $\chi\left(e_{1}\right)=\chi(w) \forall \chi \in X(A)$ and hence $\phi\left(e_{1}\right)=\phi(w) \forall \phi \in \operatorname{Mult}(A, \|$. $\|)$ because, by Theorem $2.4 \operatorname{Mult}_{m}(A, \|$. \|) is dense in $\operatorname{Mult}(A,\|\|$.$) . The unicity of e_{1}$ follows from Proposition 2.10. Similarly, there exists a unique idempotent $e_{2} \in A$ such that $\phi\left(e_{2}\right)=1 \forall \phi \in U_{2}$ and $\phi\left(e_{2}\right)=0 \forall \phi \in U_{1}$.

Definition and notations: We will denote by $|\cdot|_{\infty}$ the Archimedean absolute value of $\mathbb{R}$. Given a unital commutative ultrametric $\mathbb{K}$-normed algebra $A$ and $\phi \in M u l t(A,\|\|),. y_{1}, \ldots y_{q} \in A$ and $\epsilon>0$, we will denote by $W\left(\phi, y_{1}, \ldots, y_{q}, \epsilon\right)$ the set of $\theta \in \operatorname{Mult}(A,\|\cdot\|)$ such that $\left|\phi\left(y_{j}\right)-\theta\left(y_{j}\right)\right|_{\infty} \leq$ $\epsilon \forall j=1, \ldots, q$. Given a unital commutative ultrametric $\mathbb{K}$-normed algebra $A$ and a subalgebra $B$, we call canonical mapping from $\operatorname{Mult}(A,\|\|$.$) to \operatorname{Mult}(B, \|$. $\|)$ the mapping $\Phi$ defined by $\Phi(\varphi)(x)=\varphi(x) \forall x \in B, \varphi \in \operatorname{Mult}(A,\|\|)$.

Proposition 2.12. [14] Let $A$ be a unital commutative ultrametric $\mathbb{K}$-Banach algebra and assume that $\operatorname{Mult}(A,\|\|$.$) admits a partition in two compact subsets U, V$. There exists a $\mathbb{K}$-affinoid algebra $B$ included in $A$, admitting for norm this of $A$, such that $M u l t(B,\|\cdot\|)$ admits a partition in two open subsets $U^{\prime}, V^{\prime}$ where the canonical mapping $\Phi$ from $\operatorname{Mult}(A, \|$. $\|)$ to $\operatorname{Mult}(B, \|$. $\|)$ satisfies $\Phi(U) \subset U^{\prime}, \Phi(V) \subset V^{\prime}$.

Proof. Since $U$ and $V$ are compact sets, we can easily define a covering of open sets $\left(O_{j}\right)_{j \in J}$ such that $O_{j} \cap V=\emptyset \forall j \in J$. From this, we can extract a finite covering $\left(U_{i}\right)_{1 \leq i \leq n}$ of $U$ where the $U_{i}$ are of the form $W\left(f_{i}, x_{i, 1}, \ldots, x_{i, m_{i}}, \epsilon_{i}\right)$ with $x_{i, j} \in A$, such that $U_{i} \cap V=\emptyset \forall i=1, \ldots, n$. Let $\widetilde{A}$ be the finite type $\mathbb{K K}$-subalgebra generated by all the $x_{i, j}, 1 \leq j \leq m_{i}, 1 \leq i \leq n$. Consider the image of $\operatorname{Mult}(A,\|\|$.$) in \operatorname{Mult}(\widetilde{A},\|\|$.$) through the mapping \Phi$ that associates to each 
$\phi \in \operatorname{Mult}(A,\|\|$.$) its restriction to \widetilde{A}$ and let $\widetilde{U}=\Phi(U), \widetilde{V}=\Phi(V)$. Then both $\widetilde{U}, \widetilde{V}$ are compact with respect to the topology of $\operatorname{Mult}(\widetilde{A},\|\cdot\|)$ and hence there exist open neighborhoods $U^{\prime}$ of $\widetilde{U}$ and $V^{\prime}$ of $\widetilde{V}$ in $\operatorname{Mult}(\widetilde{A},\|\|$.$) such that U^{\prime} \cap V^{\prime}=\emptyset$. Let $Y=U^{\prime} \cup V^{\prime}$. By construction we have $\Phi(U) \subset U^{\prime}, \Phi(V) \subset V^{\prime}$.

Let $\phi \in \operatorname{Mult}(\widetilde{A},\|\|.) \backslash Y$. There exists a finite type algebra $\widetilde{A}_{\phi}$ containing $\widetilde{A}$, such that the canonical image $H_{\varphi}$ of $\operatorname{Mult}\left(\widetilde{A}_{\phi}, \|\right.$. \|) in $\operatorname{Mult}(\widetilde{A},\|\|$.$) does not contain \phi$. Since this image $H_{\phi}$ is compact, there exists a neighborhood $G(\phi)$ of $\phi$ such that $G(\phi) \cap H_{\phi}=\emptyset$. Next, we notice that $\operatorname{Mult}(\widetilde{A},\|\|.) \backslash Y$ is compact, hence we can find $\phi_{1}, \ldots, \phi_{n} \in \operatorname{Mult}(\widetilde{A}, \|$. $\|) \backslash Y$ and neighborhoods $Z\left(\phi_{1}\right), \ldots, Z\left(\phi_{n}\right)$ making a covering of $\operatorname{Mult}(\widetilde{A},\|\|.) \backslash Y$. Let $E$ be the finite type algebra generated by the $\widetilde{A}_{\phi_{i}}, 1 \leq i \leq n$. Then $E$ is a $\mathbb{K}$-subalgebra of $A$ of finite type which contains $\widetilde{A}$ and hence is equipped with the $\mathbb{K}$-algebra norm $\|$. \| of $A$. Moreover, by construction, $\operatorname{Mult}(E,\|\cdot\|)$ is equal to $Y=U^{\prime} \cup V^{\prime}$.

Let $\left\{x_{1}, \ldots, x_{N}\right\}$ be a finite subset of the unit ball of $E$ such that $\mathbb{K}\left[x_{1}, \ldots, x_{N}\right]=E$. Let $T$ be the topologically pure extension $\mathbb{K}\left\{X_{1}, \ldots, X_{N}\right\}$ and consider the canonical morphism $\Theta$ from $\mathbb{K}\left[X_{1}, \ldots, X_{N}\right]$ equipped with the Gauss norm, into $E$, equipped with the norm $\|$. $\|$ of $A$, defined as $\Theta\left(F\left(X_{1}, \ldots, X_{N}\right)\right)=F\left(x_{1}, \ldots, x_{N}\right)$. Since by hypotheses, $\left\|x_{j}\right\| \leq 1 \forall j=1, \ldots, N, \Theta$ is continuous and has expansion to a continuous morphism $\bar{\Theta}$ from $T$ into $A$. Let $\mathcal{I}$ be the closed ideal of the elements $F \in T$ such that $\bar{\Theta}(F)=0$. Then $\bar{\Theta}(T)$ is the $\mathbb{K}$-affinoid algebra $B=\frac{T}{\mathcal{I}}$ containing $E$ and included in $A$. By construction, the $\mathbb{K}$-affinoid norm of $B$ is the restriction of the norm $\|\cdot\|$ of $A$. Since by construction $E$ is dense in $B$, we have $\operatorname{Mult}(B,\|\cdot\|)=\operatorname{Mult}(E,\|\cdot\|)=U^{\prime} \cup V^{\prime}$. Consequently, $\Phi(U) \subset U^{\prime}, \Phi(V) \subset V^{\prime}$, which ends the proof.

Remark 2.13. Proposition 2.12 was roughly stated in [14]. However, its proof was confusing about subsets containing $U$ and $V$ and norms defined on an affinoid subalgebra $B$, which then puts in doubt the conclusion.

We can now conclude.

Proof of Theorem 1.1. By Proposition 2.12, there exists a $\mathbb{K}$-affinoid algebra $B$ included in $A$ such that $\operatorname{Mult}(B,\|\|$.$) admits a partition in two open disjoint subsets U^{\prime}, V^{\prime}$ and such that the canonical mapping $\Phi$ from $\operatorname{Mult}(A,\|\|$.$) to \operatorname{Mult}(B,\|\|$.$) satisfies \Phi(U) \subset U^{\prime}, \Phi(V) \subset V^{\prime}$. Now, by Proposition 2.11, there exist idempotents $u^{\prime}, v^{\prime} \in B$ such that $\phi\left(u^{\prime}\right)=1 \forall \phi \in U^{\prime}$ and $\phi\left(u^{\prime}\right)=0 \forall \phi \in V^{\prime}$. Consequently, we have $\phi(u)=1 \forall \phi \in U, \phi(u)=0 \forall \phi \in V$ and $\phi(v)=0 \forall \phi \in U$, $\phi(v)=1 \forall \phi \in V$. The unicity follows from Proposition 2.11. That ends the proof.

Proof of Theorem 1.3. Without loss of generality, we can suppose $a=0$. Let $U=\{\phi \in$ $\operatorname{Mult}(A,\|\cdot\|)\}$ such that $\phi(x) \leq r$, and let $V=\{\phi \in \operatorname{Mult}(A,\|\cdot\|)\}$ such that $\phi(x) \geq s$. Since $\operatorname{Mult}_{m}(A,\|\cdot\|)$ is dense in $\operatorname{Mult}(A,\|\cdot\|)$, it is clear that no $\phi \in \operatorname{Mult}(A,\|\cdot\|)$ can satisfy $r<\phi(x)<s$. Consequently, $U, V$ make a partition of $\operatorname{Mult}(A, \|$. $\|)$. Next, one can easily 
check that $U$ and $V$ are open and closed with respect to the pointwise convergence. Indeed, given $\phi \in \operatorname{Mult}(A,\|\cdot\|), g_{1}, \ldots, g_{t} \in A$ and $\epsilon>0$, we denote by $W\left(\phi, g_{1}, \ldots, g_{t}, \epsilon\right)$ the neighborhood of $\phi$ defined as $\left\{\theta \in \operatorname{Mult}(A,\|\|.)\left|\phi\left(g_{j}\right)-\theta\left(g_{j}\right)\right|_{\infty} \leq \epsilon \forall j=1, \ldots, t\right\}$. So, let $\left.\epsilon \in\right] 0, \frac{s-r}{2}[$ and consider the families of neighborhoods of $U$ and $V$ of the form $W\left(\phi, x, f_{1}, \ldots, f_{m}, \epsilon\right)_{\phi \in U}$ and $W\left(\psi, x, g_{1}, \ldots, g_{n}, \epsilon\right)_{\psi \in V}$ respectively. Then given any $W\left(\phi, x, f_{1}, \ldots, f_{m}, \epsilon\right), \phi \in U$ and $W\left(\psi, x, g_{1}, \ldots, g_{n}, \epsilon\right), \psi \in V$ we have $W\left(\phi, x, f_{1}, \ldots, f_{m}, \epsilon\right) \cap W\left(\psi, x, g_{1}, \ldots, g_{n}, \epsilon\right)=\emptyset$ hence $U$ and $V$ are two open subsets such that $U \cap V=\emptyset$. By construction $\operatorname{Mult}(A,\|\cdot\|)=U \cup V$. Consequently, $U$ and $V$ are two open subsets making a partition of $M u l t(A,\|\|$.$) , which by Theorem$ 1.1 , ends the proof.

Remark 2.14. The proof of Theorem 1.3 consists of injecting the Krasner-Tae algebra [8] $H(\Gamma(a, r, s))$ into $A$.

Acknowledgement: I am grateful to the Referee for useful remarks. 


\section{References}

[1] J. Araujo, Prime and maximal ideals in the spectrum of the ultrametric algebra, Contemporary of the AMS, vol. 704, 2018.

[2] V. Berkovich, Spectral Theory and Analytic Geometry over Non-Archimedean Fields, AMS Survey and Monographs, vol. 33, 1990.

[3] M. Chicourrat, and A. Escassut, "Banach algebras of ultrametric Lipschitzian functions", Sarajevo Journal of Mathematics, vol. 14, no. 2, pp. 1-12, 2018. (27)

[4] M. Chicourrat, B. Diarra, and A. Escassut, "Finite codimensional maximal ideals in subalgebras of ultrametric uniformly continuous functions", Bulletin of the Belgium Mathematical Society, vol. 26, no. 3, pp. 413-420, 2019.

[5] M. Chicourrat, and A. Escassut, "Ultrafilters and ultrametric Banach algebras of Lipschitzian functions", Advances in Operator Theory, vol. 5, no. 1, pp. 115-142, 2020.

[6] M. Chicourrat, and A. Escassut, "A survey and new results on Banach algebras of ultrametric functions", p-adic Numbers, Ultrametic Analysis and Applications, vol. 12, no. 3, pp. 185-202, 2020.

[7] A. Escassut, Analytic elements in p-adic analysis, World Scientific Publishing, 1995.

[8] A. Escassut, "Algèbres de Banach ultramétriques et algèbres de Krasner-Tate", Astérisque, no. 10, pp. 1-107, 1973.

[9] A. Escassut, Ultrametric Banach algebras, World Scientific Publishing, 2003.

[10] A. Escassut, and N. Mainetti, "Spectrum of ultrametric Banach algebras of strictly differentiable functions", t Contemporary Mathematics, vol. 704, pp. 139-160, 2018.

[11] A. Escassut, "Survey on the Kakutani problem in $p$-adic analysis I", Sarajevo Journal of Mathematics, vol. 15, no. 2, pp. 245-263, 2019.

[12] A. Escassut, "Survey on the Kakutani problem in $p$-adic analysis II", Sarajevo Journal of Mathematics, vol. 16, no. 1, pp. 55-70, 2020.

[13] B. Guennebaud, "Algèbres localement convexes sur les corps valués", Bulletin des Sciences Mathématiques, vol. 91, pp. 75-96, 1967.

[14] B. Guennebaud, Sur une notion de spectre pour les algèbres normées ultramétriques, Thèse d'Etat, Université de Poitiers, 1973. 
[15] Ch. E. Rickart, General Theory of Banach Algebras, Krieger Publishing Company, 2002.

[16] P. Salmon, "Sur les séries formelles restrintes", Bulletin de la Société Mathématique de France, vol. 92, pp. 385-410, 1964.

[17] J. Tate, "Rigid analytic spaces", Inventiones Mathematicae, vol. 12, pp. 257-289, 1971. 